\title{
Skin autofluorescence: early sign of lung function deterioration?
}

\author{
Emiel F.M. Wouters ${ }^{1,2}$ and Niki L. Reynaert ${ }^{1}$ \\ ${ }^{1}$ Dept of Respiratory Medicine, NUTRIM School of Nutrition and Translational Research in Metabolism, \\ Maastricht University Medical Center, Maastricht University, Maastricht, The Netherlands. ${ }^{2}$ Ludwig Boltzmann \\ Institute for Lung Health, Vienna, Austria.
}

Correspondence: Niki L. Reynaert, Dept of Respiratory Medicine, Maastricht University, P0 Box 616, 6200MD Maastricht, The Netherlands. E-mail: n.reynaert@maastrichtuniversity.nl

@ERSpublications

The relationship between SAF and $\mathrm{FEV}_{1}, D_{\mathrm{LCO}}$ and $X_{5}$, independent of COPD or diabetes. Will individuals with raised SAF and subclinical lung function alterations develop disease? What mechanisms underlie parenchymal alterations indicated by SAF? https://bit.ly/2YTtdkL

Cite this article as: Wouters EFM, Reynaert NL. Skin autofluorescence: early sign of lung function deterioration? ERJ Open Res 2020; 6: 00368-2020 [https://doi.org/10.1183/23120541.00368-2020].

Advanced glycation end products (AGEs) comprise a heterogeneous group of stable end-products of the non-enzymatic glycation reactions described by Maillard in 1912, in which reduced sugars irreparably modify proteins, lipids and DNA. This is a slow physiological process and accumulation of AGEs is part of normal ageing. Under conditions of hyperglycaemia and oxidative stress, a much faster and more substantial generation of AGEs is induced. The myriad of pathogenic signals AGEs can induce and the fact that enhanced AGE accumulation accompanies a broad spectrum of age-associated chronic inflammatory diseases [1] form the basis of the Maillard theory of ageing [2].

A number of AGEs are characterised by their yellow-brown fluorescent colour and their ability to form stable molecular crosslinks on long-lived proteins such as skin collagens $[3,4]$. The skin offers an excellent opportunity for noninvasive investigation of AGE accumulation, through their auto-fluorescent properties [5]. As such, glycation-associated skin autofluorescence (SAF) correlates with chronological ageing in healthy subjects [6] and enhanced accumulation of AGEs in the skin has been detected in diabetes $[4,6]$. In the search for (noninvasive) biomarkers for COPD, studies have shown increased SAF in COPD as well, which inversely correlated with indices of lung function. In the current issue of ERJ Open Research, the study by ZaIgham et al. [7] is only the second to extend these findings into a population-based study, of mostly 50-64-year-olds.

Here, ZAIGHAM et al. [7] examined the relationship between SAF and detailed lung function measures using impulse oscillometry (IOS) and diffusing capacity in the population-based cohort SCAPIS (Swedish CArdioPulmonary bioImage Study). Subjects with SAF in the highest tertile were generally older, more likely to be current smokers, and represented a larger proportion of patients with COPD and diabetes, reflected by higher mean values of $\mathrm{HbAlc}$, and lower mean values of spirometry and diffusing capacity. When correcting for confounders, or restricting the analyses to nonsmokers or subjects without COPD, inverse relationships between SAF and forced expiratory volume in $1 \mathrm{~s}\left(\mathrm{FEV}_{1}\right)$, forced vital capacity (FVC), diffusing capacity of the lung for carbon monoxide $\left(D_{\mathrm{LCO}}\right)$ and DLCO/alveolar volume remained significant. From the IOS data, it appeared that SAF was especially related to respiratory reactance, namely as an inverse correlation with reactance measured at $5 \mathrm{~Hz}$. This relationship was again also apparent in non-COPD subjects, but absent in nonsmokers. The association with $\mathrm{FEV}_{1}$ has been shown in previous studies in patients with COPD [8-10], whereas the loss of a significant relationship between SAF and the

Copyright $\odot$ ERS 2020. This article is open access and distributed under the terms of the Creative Commons Attribution Non-Commercial Licence 4.0. 
$\mathrm{FEV}_{1} / \mathrm{FVC}$ ratio after correcting for multiple confounders is in contrast to previous reports in healthy patients and COPD patients $[10,11]$.

Based on the observed association of SAF with $D_{\mathrm{LCO}}$, the authors speculate about relationships to changes in the lung parenchyma, either emphysematous or fibrotic in nature. The diffusing capacity is determined by two components, the alveolar-capillary membrane diffusing capacity and the pulmonary capillary blood volume [12]. Previous studies in diabetes indicated that a reduction in alveolar-capillary membrane diffusing capacity can be related to thickening of the pulmonary capillary basal lamina or increases in endothelial permeability, resulting in an overall reduction in $D_{\text {LCO }}$ [13-15]. Pulmonary capillary blood volume is determined by the number of pulmonary capillaries in contact with ventilated alveoli, which is also reduced in conditions such as diabetes [16]. This subclinical attenuation of lung function, reflected in a restrictive spirometric pattern and impaired diffusing capacity, is well recognised in diabetics $[13,14]$. Moreover, already at a pre-diabetes stage, negative effects on lung function have been reported $[17,18]$. The impaired lung function in diabetes is associated with glycaemic control and disease duration, both of which are associated with increased AGE levels [18-20]. The non-enzymatic glycation of pulmonary extracellular matrix components is often proposed as the mechanism underlying the negative effects of hyperglycaemia on lung function, but this has never been examined. Moreover, although enhanced SAF in diabetics has been associated with (micro)-vascular complications [21], it has not been examined in relation to lung function. It seems plausible to link the accumulation of AGEs to these intermediary processes rather than to specific parenchymal abnormalities (destructive or fibrotic).

The authors hypothesise that the reported inverse association between SAF and $\mathrm{X}_{5}$ could be linked to changes in elastance of the respiratory system. However, data obtained by oscillometry and pulmonary mechanical resonance imaging clearly indicate that increased ventilation heterogeneity in never-smokers and ex-smokers without COPD did not reflect in qualitatively apparent changes in oscillometry. Therefore, this isolated relationship between $\mathrm{X}_{5}$ and $\mathrm{SAF}$ must be cautiously interpreted in terms of underlying airway and parenchymal biomechanical abnormalities [22]. A relationship with emphysema is assumed based on its strong associations with the receptor for AGEs (RAGE). However, it is of interest to note that AGEs elicit more broad pathogenic effects than solely through activation of RAGE. AGEs can indeed directly impact the stiffness of the extracellular matrix, as well as disturb matrix turnover in favour of collagen deposition [23].

It remains unclear to what extent SAF serves as a proxy for AGEs in general. AGEs are formed through various sequential chemical reactions and consist of a broad range of compounds. Because not all AGEs have auto-fluorescent properties, their measurement through autofluorescence only represents a portion of them.

It is poorly understood if and how SAF relates to AGE accumulation and effects thereof in other organs, including in disease. To minimise external influences, such as from UV light, it is recommended to measure SAF on the volar side of the arm. Cigarette smoke accelerates skin ageing and contains glycotoxins that induce AGE formation [24]. Enhanced SAF has indeed been shown in smokers compared to nonsmokers [25, 26], as did the study by ZaIGHAM et al. [7]. Some studies particularly measured SAF in the non-dominant arm to limit external effects of smoking. Direct effects of smoke are therefore less likely to underlie SAF, but cannot be ruled out.

AGEs transported through the circulation are the most attractive connection between SAF and other organs. Elevated circulating AGE levels are a result of imbalanced uptake through the diet, intake via smoking and endogenous production versus elimination. In various chronic inflammatory conditions enhanced circulating AGE levels were measured, but very few studies investigated associations between SAF and circulating AGEs. When we examined the association between three different plasma AGEs and SAF, surprisingly only significant relationships with the non-fluorescent AGEs N(6)-carboxymethyllysine $(\mathrm{CML})$ and $\mathrm{Ne}$-(carboxyethyl)lysine (CEL) were observed. For plasma CML, which decreased in COPD patients, this was even an inverse association. One study reported on the differential accumulation in skin, plasma and lung tissue in COPD patients. Hoonhorst et al. [9] found increased SAF, but no evidence of enhanced levels of AGEs in plasma, sputum or bronchial biopsies. This is in contrast to earlier reports of increased plasma CEL levels and accumulation of AGEs in lungs of COPD patients, which both negatively correlated with lung function $[27,28]$. Also in the lining fluid of COPD patients, CML was increased compared to healthy ex- and current smokers [28].

These discrepancies can likely be attributed to the various methods employed to measure AGEs. Mass spectrometry is the most sensitive, specific and reliable method to measure AGEs. Commercially available antibody-based assays are more commonly used, but lack specificity. Finally, fluorescence-based assays only capture a fraction of AGEs. 
In general, the interpretation of AGE measures or proxy's thereof across different organs is complicated by the complex chemistry behind AGE formation, the multiple sites of origin, the variable turnover rate of targeted proteins and equilibrium between formation and elimination.

Regardless, enhanced levels of circulating AGEs represent a potential common underlying mechanism of multi-organ and multi-morbid conditions that are often observed in the elderly, in which vascular effects are a common denominator. Diabetes and its complications form the basis for this theory. Moreover, data support the role for AGEs in the onset and progression of cardiovascular diseases through different mechanisms including direct effects on vascular stiffness, pro-inflammatory effects, endothelial dysfunction and pro-coagulant activity $[29,30]$. Interestingly, in COPD enhanced AGE presence has been seen in endothelial cells of both the lungs and the kidneys, which was linked to endothelial damage [31]. Within this theory, there might be an opportunity to use SAF as an early biomarker, as it has been associated with the individual conditions, but follow-up examinations are needed to look into specific organs affected.

With respect to the lungs and their functioning, IOS is a sensitive method and is able to detect more subtle changes when compared to standard spirometry. As such, it could be more useful to establish an earlier diagnosis or monitor more subtle changes over time. As stated by the authors, it would be of great interest to examine whether individuals with raised SAF and subclinical lung function alterations determined by IOS and/or diffusing capacity will go on to develop disease. Furthermore, it would be important to confirm these IOS abnormalities and their possible association with SAF in other cohorts.

Conflict of interest: E.F.M. Wouters has nothing to disclose. N.L. Reynaert has nothing to disclose.

\section{Reference}

1 Reynaert NL, Gopal P, Rutten EPA, et al. Advanced glycation end products and their receptor in age-related, non-communicable chronic inflammatory diseases; overview of clinical evidence and potential contributions to disease. Int J Biochem Cell Biol 2016; 81: 403-418.

2 Monnier VM. Nonenzymatic glycosylation, the Maillard reaction and the aging process. J Gerontol 1990; 45: B105-B111.

3 Bierhaus A, Hofmann MA, Ziegler R, et al. AGEs and their interaction with AGE-receptors in vascular disease and diabetes mellitus. I. The AGE concept. Cardiovasc Res 1998; 37: 586-600.

4 Monnier VM, Kohn RR, Cerami A. Accelerated age-related browning of human collagen in diabetes mellitus. Proc Natl Acad Sci USA 1984; 81: 583-587.

5 Meerwaldt R, Hartog JW, Graaff R, et al. Skin autofluorescence, a measure of cumulative metabolic stress and advanced glycation end products, predicts mortality in hemodialysis patients. J Am Soc Nephrol 2005; 16: 3687-3693.

6 Dyer DG, Dunn JA, Thorpe SR, et al. Accumulation of Maillard reaction products in skin collagen in diabetes and aging. J Clin Invest 1993; 91: 2463-2469.

7 Zaigham S, Persson M, Jujic A, et al. Measures of lung function and their relationship with advanced glycation end-products. ERJ Open Res 2020; 6: 00356-2019.

8 Gopal P, Reynaert NL, Scheijen JL, et al. Plasma advanced glycation end-products and skin autofluorescence are increased in COPD. Eur Respir J 2014; 43: 430-438.

9 Hoonhorst SJ, Loi AT, Pouwels SD, et al. Advanced glycation endproducts and their receptor in different body compartments in COPD. Respir Res 2016; 17: 46.

10 Hoonhorst SJ, Loi AT, Hartman JE, et al. Advanced glycation end products in the skin are enhanced in COPD. Metab Clin Exp 2014; 63: 1149-1156.

11 Kubo A, Kato M, Sugioka Y, et al. Relationship between advanced glycation end-product accumulation in the skin and pulmonary function. J Phys Ther Sci 2018; 30: 413-418.

12 Roughton FJ, Forster RE. Relative importance of diffusion and chemical reaction rates in determining rate of exchange of gases in the human lung, with special reference to true diffusing capacity of pulmonary membrane and volume of blood in the lung capillaries. J Appl Physiol 1957; 11: 290-302.

13 Vracko R, Thorning D, Huang TW. Basal lamina of alveolar epithelium and capillaries: quantitative changes with aging and in diabetes mellitus. Am Rev Respir Dis 1979; 120: 973-983.

14 Weynand B, Jonckheere A, Frans A, et al. Diabetes mellitus induces a thickening of the pulmonary basal lamina. Respiration 1999; 66: 14-19.

15 Popov D, Hasu M, Costache G, et al. Capillary and aortic endothelia interact in situ with nonenzymatically glycated albumin and develop specific alterations in early experimental diabetes. Acta Diabetol 1997; 34: 285-293.

16 Niranjan V, McBrayer DG, Ramirez LC, et al. Glycemic control and cardiopulmonary function in patients with insulin-dependent diabetes mellitus. Am J Med 1997; 103: 504-513.

17 Sanchez E, Gutierrez-Carrasquilla L, Barbe F, et al. Lung function measurements in the prediabetes stage: data from the ILERVAS Project. Acta Diabetol 2019; 56: 1005-1012.

18 Lee MJ, Coast JR, Hempleman SC, et al. Type 1 diabetes duration decreases pulmonary diffusing capacity during exercise. Respiration 2016; 91: 164-170.

19 Yeh HC, Punjabi NM, Wang NY, et al. Cross-sectional and prospective study of lung function in adults with type 2 diabetes: the Atherosclerosis Risk in Communities (ARIC) study. Diabetes Care 2008; 31: 741-746.

20 Walter RE, Beiser A, Givelber RJ, et al. Association between glycemic state and lung function: the Framingham Heart Study. Am J Respir Crit Care Med 2003; 167: 911-916.

21 Monnier VM, Sell DR, Genuth S. Glycation products as markers and predictors of the progression of diabetic complications. Ann N Y Acad Sci 2005; 1043: 567-581. 
Eddy RL, Westcott A, Maksym GN, et al. Oscillometry and pulmonary magnetic resonance imaging in asthma and COPD. Physiol Rep 2019; 7: e13955.

23 Monnier VM, Mustata GT, Biemel KL, et al. Cross-linking of the extracellular matrix by the maillard reaction in aging and diabetes: an update on "a puzzle nearing resolution". Ann N Y Acad Sci 2005; 1043: 533-544.

24 Cerami C, Founds H, Nicholl I, et al. Tobacco smoke is a source of toxic reactive glycation products. Proc Natl Acad Sci USA 1997; 94: 13915-13920.

25 Koetsier M, Lutgers HL, de Jonge C, et al. Reference values of skin autofluorescence. Diabetes Technol Ther 2010; 12: 399-403.

26 Lutgers HL, Graaff R, Links TP, et al. Skin autofluorescence as a noninvasive marker of vascular damage in patients with type 2 diabetes. Diabetes Care 2006; 29: 2654-2659.

27 Wu L, Ma L, Nicholson LF, et al. Advanced glycation end products and its receptor (RAGE) are increased in patients with COPD. Respir Med 2011; 105: 329-336.

28 Kanazawa H, Kodama T, Asai K, et al. Increased levels of N(epsilon)-(carboxymethyl)lysine in epithelial lining fluid from peripheral airways in patients with chronic obstructive pulmonary disease: a pilot study. Clin Sci 2010; 119: 143-149.

29 Fishman SL, Sonmez H, Basman C, et al. The role of advanced glycation end-products in the development of coronary artery disease in patients with and without diabetes mellitus: a review. Mol Med 2018; $24: 59$.

30 Prasad A, Bekker P, Tsimikas S. Advanced glycation end products and diabetic cardiovascular disease. Cardiol Rev 2012; 20: 177-183.

31 Polverino F, Laucho-Contreras ME, Petersen $\mathrm{H}$, et al. A pilot study linking endothelial injury in lungs and kidneys in chronic obstructive pulmonary disease. Am J Respir Crit Care Med 2017; 195: 1464-1476. 